\title{
Ultrafast spectroscopy of UV-induced DNA-lesions - on the search for strategies which keep DNA alive
}

\author{
W. Zinth ${ }^{1}$, B. P. Fingerhut ${ }^{2}$, T. T. Herzog ${ }^{1}$, G. R. Ryseck ${ }^{1,3}$, K. Haiser ${ }^{1}$, F. F. Graupner ${ }^{1}$, K. Heil $^{4}$, \\ P. Gilch ${ }^{1,3}$, W. J. Schreier ${ }^{1}$, T. Carell ${ }^{4}$, R. de Vivie-Riedle ${ }^{2}$ \\ ${ }^{1}$ Faculty of Physics, Ludwig Maximilians University Munich, 80538 München, Germany \\ ${ }^{2}$ Department of Chemistry, Ludwig Maximilians University Munich, 81377 München, Germany \\ ${ }^{3}$ Institut für Physikalische Chemie, Heinrich-Heine-Universität Düsseldorf, 40225 Düsseldorf, \\ Germany \\ ${ }^{4}$ Department of Chemistry, Center for Integrative Protein Science, Ludwig Maximilians University \\ Munich, 81377 München, Germany
}

\begin{abstract}
UV-induced photolesions are studied in the visible and IR. While structural distortions of the DNA-backbone at the moment of light absorption are prerequisite for CPD-formation, strain from the backbone guides Dewar-formation.
\end{abstract}

\section{Introduction}

Ultraviolet (UV) radiation induces a series of photo-damages in DNA which can lead to mutations and skin cancer [1]. These lesions occur predominantly at DNA sites where two pyrimidine bases, especially thymines (T) are adjacent in the DNA strand. Among the different UVlesions the CPD (cyclobutane pyrimidine dimer) photolesion is the most frequent, followed by the $\mathrm{T}(6-4) \mathrm{T}$ and the Dewar valence isomer (structures are depicted in Fig. 1). In this contribution we use time resolved UV-pump, IR/vis probe-spectroscopy and quantum chemistry to study the formation of the CPD lesion and the Dewar photoproduct. We present results from different model systems (dinucleotides, dimers and single strands). The experiments reveal that the formation of the CPD lesion occurs within $\sim 1 \mathrm{ps}$, i. e. during the ultra-rapid decay of the excited singlet state [2-6]. For this reaction a favourable arrangement of the adjacent thymine molecules is a prerequisite and variations of the arrangement influence the quantum efficiency $[4,6]$. The secondary photoreaction from the $\mathrm{T}(6-4) \mathrm{T}$ lesion to the Dewar isomer occurs on the $100 \mathrm{ps}$ time scale [7]. By the combination of different experimental techniques and theoretical simulations we obtain deep insight into the excited state dynamics of Dewar formation. Here the molecules are always in an arrangement susceptible to Dewar formation. However it is the connection of two thymine residues and the strain induced by the DNA backbone which promotes Dewar formation.

The different molecular structures and reaction schemes are shown in Fig. 1 (left). Illumination of a pair of thymine bases (top, left) in the absorption band peaking around $260 \mathrm{~nm}$ (Fig. 1, right), i. e. in the spectral range with very low solar irradiation, populates the excited $\pi \pi^{*}$ state of thymine which decays on the time scale of a few hundred fs [2 - 4]. We could show recently that the formation of the CPD lesion occurs on the same time scale $[5,6]$. The CPD and the T(6-4)T photolesions are primary products resulting from the absorption of one photon in the UV-C range and have very low formation yields in the range of ca. (CPD) $1 \%$ and even below (T(6-4)T). On the 
other hand, the Dewar valence isomer is a photolesion which is formed in a secondary absorption process from T(6-4)T with a comparably high yield (8\%) [7]. Since T(6-4)T has an absorption band which is red-shifted into a spectral range of UV-A with considerable solar irradiance at sea level, the Dewar lesion can be formed in significant amounts. The experimental characterization of the reaction dynamics of CPD and Dewar formation will be presented together with a theoretical modelling giving a detailed insight into the molecular principles leading to these DNA photolesions.
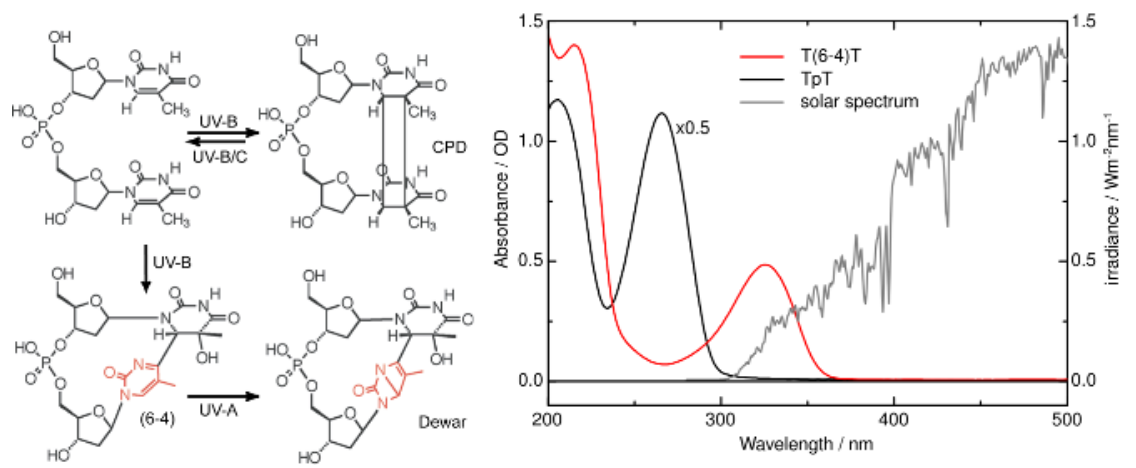

Fig. 1. Structures of different photolesions after UV excitation of two neighbouring thymine residues (left). Absorption spectra of TMP and the T(6-4)T photolesion. The latter shows a red-shifted absorption in the UV-A range where solar emission (at sea level) starts to be significant (right).

\section{Results and discussion}

The synthesis of the different investigated DNA samples has been presented in [5, 7] . Details on the time resolved techniques are given in the literature [5, 7]. In brief: Time-resolved infrared spectra were recorded using a pump-probe set-up based on a commercial Ti:sapphire laser amplifier system (Spectra Physics, Spitfire Pro) combined with a NOPA and IR generation. Fluorescence emission from the excited electronic state is recorded by a streak-camera (Hamamatsu C5680-24 C).

The formation of the CPD-lesion is studied for model compounds with different relative arrangements of the thymine bases. The CPD lesion can be identified via three marker bands in the mid-infrared and the dynamics of CPD formation was observed via probing with fs IR pulses. All investigated compounds show a very rapid rise of the marker bands on the time scale of below $1 \mathrm{ps,}$ i. e. with the decay of the originally excited electronic $\pi \pi^{*}$ state. Additional studies covering the nano- and microsecond range gave no indication for CPD formation at later times. The high speed of CPD formation shows that the relative arrangement of the involved thymine molecules in the moment of light absorption determines if the CPD lesion is formed or not. This finding is supported by the observation, that the formation yield depends strongly on details in the DNA structure. Thus, the native structure of the double helix determines the low reaction yield. For the few favourably arranged thymines however the reaction rate is extremely high. The often discussed triplet states do not contribute significantly to lesion formation. This will be addressed in a forthcoming publication.

Excitation of the $\mathrm{T}(6-4) \mathrm{T}$ in the $320 \mathrm{~nm}$ range leads to population of the excited electronic $\mathrm{S}_{1}$ $\left(\pi \pi^{*}\right)$ state which can be monitored via its fluorescence emission by a streak-camera (see Fig. 2, left). The fluorescence decays predominantly on the $100 \mathrm{ps}$ time scale. A minor fraction $(\sim 10 \%)$ exists with a lifetime of ca. $1 \mathrm{~ns}$, which will not be considered here. Interestingly the time constant of the fluorescence decay is temperature dependent. An Arrhenius analysis shows that the reaction has an activation barrier of $\sim 1100 \mathrm{~cm}^{-1}$. The formation of the Dewar lesion can be followed in the IR via its prominent marker band at $1780 \mathrm{~cm}^{-1}$. The absorption of this band (see Fig. 2, right) rises on the 100 ps time scale, i. e. the Dewar lesion is formed with the decay of the excited $\pi \pi^{*}$ state.

The theoretical description of these reaction dynamics reveals interesting molecular features. After optical excitation a first motion leads the system from the Franck-Condon region to the 
minimum on the excited state potential energy surface. This process (time constant $2.5 \mathrm{ps}$ ) is well seen in transient absorption experiments. Since the reactive parts of the molecule are prearranged in the T(6-4)T starting compound no large-scale structural changes happen. It is only after the crossing of the potential barrier that specific structural aspects become important. The simulation shows that Dewar-formation can only take place if this part of the reaction is guided towards the reactive conical intersection. The guidance occurs via the strain imposed by the backbone part between the two thymines. For model compounds without backbone no Dewar formation is observed $[7,8]$.
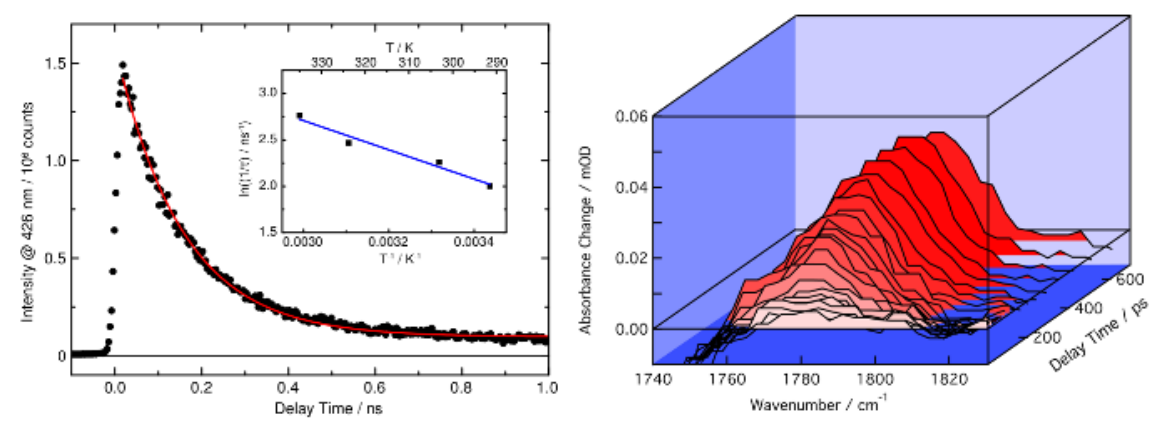

Fig. 2. UV induced emission of $\mathrm{T}(6-4) \mathrm{T}$ recorded by a streak camera (left). Transient IR absorption spectra recorded in the range of the Dewar marker band around $1780 \mathrm{~cm}^{-1}$.

\section{Conclusion}

Time-resolved experiments on the formation of two important DNA photolesions have clearly shown that the DNA structure strongly influences lesion formation. For CPD formation where the lesion is only created from the excited electronic $S_{1}$ state, the rapid decay of this state requires an appropriate structural pre-arrangement. The DNA double helix has a structure where this "dangerous" arrangement is rare and where the yield for the CPD-lesion becomes low. On the other hand, the present investigations show that Dewar lesion is only formed when the DNA backbone is present. However, since the linear arrangement of information bits in the DNA depends on the covalent connection of adjacent nuclear acids, Nature had to use other strategies to preserve the integrity of DNA.

\section{References}

1. M. G. Friedel, M. K. Cichon, T. Carell, in CRC Handbook of Organic Photochemistry and Photobiology, W. Horspool, F. Lenci, Eds. (CRC Press LLC, 2004), pp. 141/1 - 141/22.

2. C. E. Crespo-Hernandez, B. Cohen, B. Kohler, Chem.Rev. 104, 1977 (2004).

3. Y. K. Law, J. Azadi, C. E. Crespo-Hernandez, E. Olmon, B. Kohler. Biophys. J. 94, 3590 (2008).

4. C. T. Middleton, K. de La Harpe, C. Su, Y. K. Law, C. E. Crespo-Hernández, B. Kohler, Annu. Rev. Phys. Chem. 60, 217 (2009).

5. W. J. Schreier, T. E. Schrader, F. O. Koller, P. Gilch, C. E. Crespo-Hernández, V. N. Swaminathan, T. Carell, W. Zinth, B. Kohler, Science 315, 625 (2007).

6. W. J. Schreier, J. Kubon, N. Regner, K. Haiser, T. E. Schrader, W. Zinth, P. Clivio, P. Gilch, J. Am. Chem. Soc. 131, 5038 (2009).

7. K. Haiser, B. P. Fingerhut, K. Heil, A. Glas, T. T. Herzog, B. M. Pilles, W. J. Schreier, W. Zinth, R. de Vivie-Riedle, T. Carell, Angew. Chem. Int. Ed. 51, 408 (2012).

8. G. Ryseck, T. Schmierer, K. Haiser, W.J. Schreier, W. Zinth, P. Gilch, ChemPhysChem 12, 1880 (2011). 